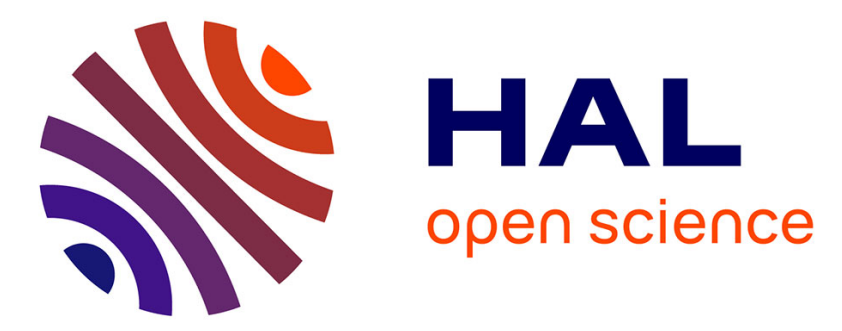

\title{
Comparison of spin photocurrent in devices based on in-plane or out-of-plane magnetized CoFeB spin detectors
}

Xiaodi Xue, Laipan Zhu, Wei Huang, Xavier Marie, Pierre Renucci, Yu Liu, Yang Zhang, Xiaolin Zeng, Jing Wu, Bo Xu, et al.

\section{To cite this version:}

Xiaodi Xue, Laipan Zhu, Wei Huang, Xavier Marie, Pierre Renucci, et al.. Comparison of spin photocurrent in devices based on in-plane or out-of-plane magnetized CoFeB spin detectors. Physical Review B: Condensed Matter and Materials Physics (1998-2015), 2019, 100, 10.1103/PhysRevB.100.045417 . hal-02388090

\section{HAL Id: hal-02388090 https://hal.science/hal-02388090}

Submitted on 1 Dec 2019

HAL is a multi-disciplinary open access archive for the deposit and dissemination of scientific research documents, whether they are published or not. The documents may come from teaching and research institutions in France or abroad, or from public or private research centers.
L'archive ouverte pluridisciplinaire HAL, est destinée au dépôt et à la diffusion de documents scientifiques de niveau recherche, publiés ou non, émanant des établissements d'enseignement et de recherche français ou étrangers, des laboratoires publics ou privés. 


\title{
Comparison of spin photocurrent in devices based on in-plane or out-of-plane magnetized CoFeB spin detectors
}

\author{
Xiaodi Xue, ${ }^{1,2}$ Laipan Zhu, ${ }^{1,3}$ Wei Huang, ${ }^{1,4}$ Xavier Marie, ${ }^{5}$ Pierre Renucci, ${ }^{5}$ Yu Liu, ${ }^{1,6}$ Yang Zhang, ${ }^{1,6}$ Xiaolin Zeng, ${ }^{1,6}$ \\ Jing Wu, ${ }^{1,6}$ Bo Xu, ${ }^{1}$ Zhanguo Wang, ${ }^{1}$ Yonghai Chen, ${ }^{1,6, *}$ Weifeng Zhang, ${ }^{2, *}$ and Yuan Lu ${ }^{7, *}$ \\ ${ }^{1}$ Key Laboratory of Semiconductor Materials Science, Institute of Semiconductors, Chinese Academy of Sciences, \\ Beijing Key Laboratory of Low Dimensional Semiconductor Materials and Devices, Beijing 100083, China \\ ${ }^{2}$ Henan Key Laboratory of Photovoltaic Materials, Laboratory of Low-Dimensional Materials Science,School of Physics \& Electronics, \\ Henan University, Kaifeng 475004, China \\ ${ }^{3}$ Beijing Institute of Nanoenergy and Nanosystems, Chinese Academy of Sciences, Beijing 100083, China \\ ${ }^{4}$ Science and Technology on Monolithic Integrated Circuits and Modules Laboratory, \\ Nanjing Electronic Devices Institute, Nanjing 210016, China \\ ${ }^{5}$ Université de Toulouse, INSA-CNRS-UPS, LPCNO, 135 Avenue de Rangueil, F-31077 Toulouse, France \\ ${ }^{6}$ Center of Materials Science and Optoelectronics Engineering, University of Chinese Academy of Sciences, Beijing 100049, China \\ ${ }^{7}$ Université de Lorraine, Institut Jean Lamour, UMR CNRS 7198, campus ARTEM, 2 Allée André Guinier, 54011, Nancy, France
}

(Received 28 November 2018; revised manuscript received 20 June 2019; published 23 July 2019)

\begin{abstract}
We have measured a helicity-dependent photocurrent at zero external magnetic field in a device based on a semiconductor quantum well embedded in a $p-i-n$ junction. The device is excited under vertical incidence with circularly polarized light. The spin filtering effect is evidenced in the temperature range $77-300 \mathrm{~K}$ owing to a $\mathrm{CoFeB} / \mathrm{MgO}$ spin filter with out-of-plane magnetization in remanence. The helicity-dependent photocurrent is explored as a function of the temperature and bias. These characteristics are compared with those of a spin photocurrent device with in-plane magnetized $\mathrm{CoFeB} / \mathrm{MgO}$ spin filter, excited under oblique incidence with circularly polarized light. In contrast to the in-plane spin filter device, the circularly polarized light asymmetry of the photocurrent in the out-of-plane device depends weakly on the external bias. The two devices are sensitive to the spin filtering of either the in-plane $\left(S_{x}\right)$ or out-of-plane $\left(S_{z}\right)$ photogenerated electron spin in the semiconductor quantum well. The helicity-dependent photocurrent results can be explained by the Dyakonov-Perel electron spin-relaxation mechanism. Our study reveals the giant spin relaxation anisotropy in III-V zinc-blende quantum wells in the presence of a vertical electric field.
\end{abstract}

DOI: 10.1103/PhysRevB.100.045417

\section{INTRODUCTION}

Semiconductor spintronic devices utilizing carriers' spin degrees of freedom provide a variety of potential applications in optics, photonics, and electronics [1-3]. Among spintronic devices, spin light emitting diode (spin-LED) has been proved to be an efficient way to transfer the solid-state information stored within ferromagnetic materials into circularly polarized photons emitted via carrier-photon angular momentum conversion [4-8]. Potential devices ranging from a memory element with optical readout and optical transport of spin information [9], advanced optical switches [10], circularly polarized single photon emitters for quantum cryptography [11] to chiral analysis [12] and three-dimensional display screens [13] are anticipated. The symmetrical challenge, which consists in an electrical detection of a spin-polarized current of carriers, is also a requirement for future spintronic devices. Some spectacular experiments have been realized with coupled electrical and optical techniques as in Refs. [14,15], where spin imaging is obtained through spatially resolved

*Corresponding authors: yhchen@semi.ac.cn; wfzhang@henu. edu.cn; yuan.lu@univ-lorraine.fr
Kerr rotation or full electrical measurements. By reversing the operation condition of spin LED, one can also realize the spin photodiode function by illuminating the device with circularly polarized light and obtain the helicity asymmetry of the photocurrent $[16,17]$. Several research groups have investigated the possibility of detecting a spin-polarized photocurrent generated under circular polarized light using spin-LED structures $[18,19]$. The spin photocurrent can be measured even at room temperature, which provides not only a powerful way to investigate the potential mechanisms of the spin relaxation but also promising applications in spin detectors of circularly polarized light.

According to the optical selection rules [20,21], a conventional spin electrode with in-plane magnetization [22-24] cannot satisfy the practical application for quantum well (QW)-based spin-LEDs, because a strong external magnetic field in the range of up to a few Tesla is required to rotate the magnetization from the in-plane to the out-of-plane direction. Under these circumstances, it is hard to distinguish the spinrelated effect from the magnetic circular dichroism of the magnetic layer and from the artificial effect due to the Zeeman splitting in the semiconductor part [25]. To our knowledge, there have been very few attempts to work at zero external magnetic field $[26,27]$. Very recently, Zhu et al. systematically 

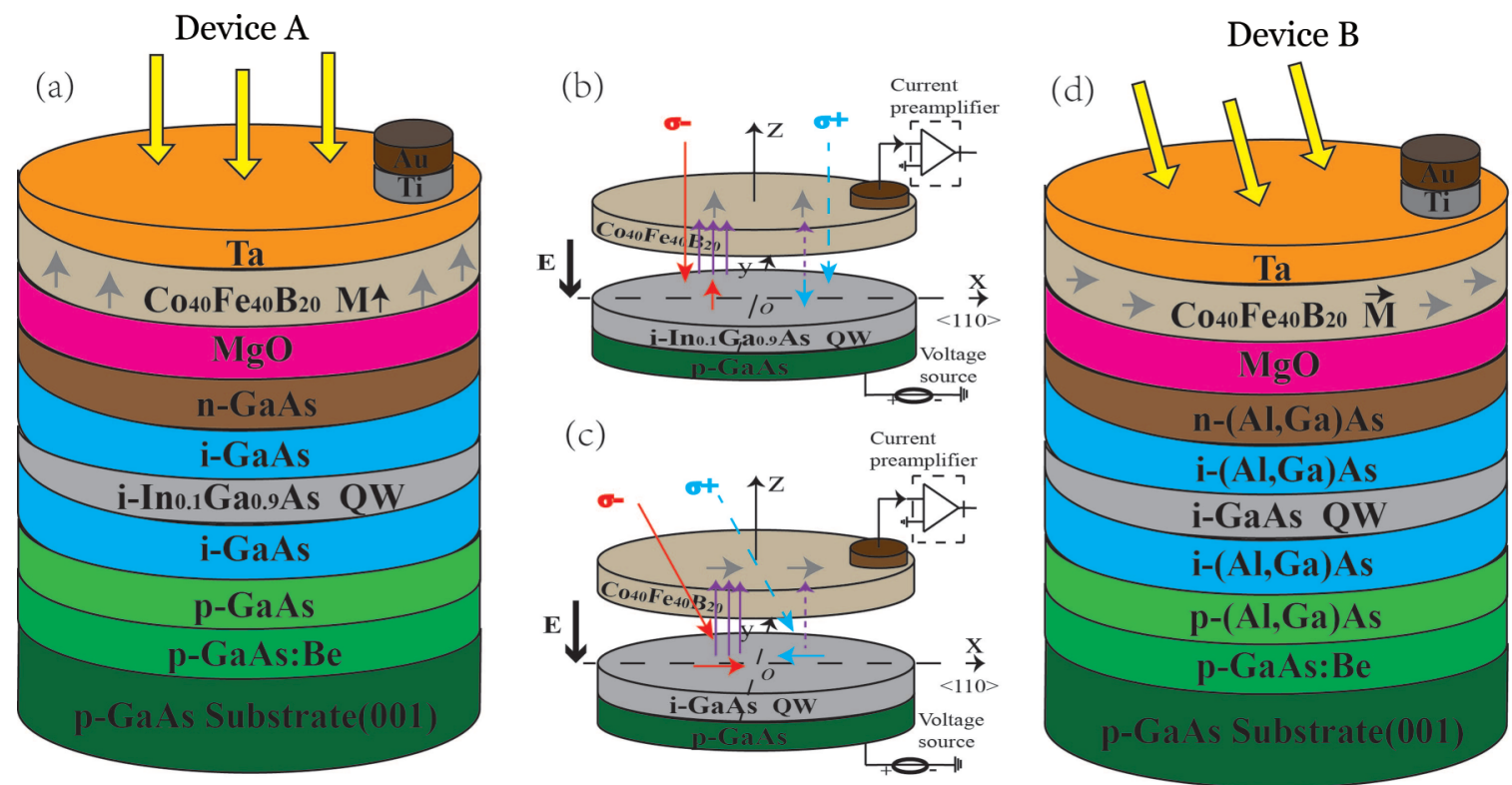

FIG. 1. Schematic diagram of the devices and photocurrent measurement. (a) out-of-plane magnetization, Device A. and (d) in-plane magnetization (Ref. [27]), Device B. The grey arrows in the CoFeB layer denote the orientation of the remnant magnetic moment. (b) Schematic of the photocurrent between right and left circularly polarized light with vertical incidence thanks to a selective spin filtering by the Co-Fe$\mathrm{B} / \mathrm{MgO}$ layer. The red and blue vertical arrows correspond to $S_{z}$, the projection along $z$ of the photogenerated average spin $\mathbf{S}$ for $\sigma^{+}$and $\sigma^{-}$, respectively (Device A). The excitation is resonant with the LH1-e1 transition. (c) Oblique incidence with $15^{\circ}$ incident angle, Device B. The red and blue horizontal arrows correspond to $S_{x}$, the projection along $x$ of the photogenerated average spin $S$ for $\sigma^{+}$and $\sigma^{-}$, respectively.

studied the influence of the incident and azimuth angles of the incoming light wave vector on the helicity asymmetry of the photocurrent without an external magnetic field based on an in-plane magnetic electrode [27]. However, systematic studies of the spin photocurrent in spin-LEDs with a perpendicularly magnetized layer are still lacking. Therefore, it was not possible to study the influence of the spin orientation on the helicity asymmetry.

In this paper, we demonstrate that the vertical illumination on a spin-LED structure with circularly polarized light at room temperature and zero magnetic field can generate a spin-polarized photocurrent by employing an out-of-plane magnetized $\mathrm{CoFeB} / \mathrm{MgO}$ electrode. The spin filtering effect is verified by studying the photocurrent asymmetry variation at different magnetic fields. Then, we investigate the influence of the external bias voltage and temperature on the circularly polarized light asymmetry of the photocurrent. The results are compared with the ones obtained on another device with in-plane magnetization and investigated in Ref. [27]. In the two devices, the spin filtering occurs with either the out-ofplane $\left(S_{z}\right)$ or in-plane $\left(S_{x}\right)$ photogenerated electron spin in the semiconductor quantum well. We interpret the different behaviors with the external bias as a consequence of the different dependence of the Dyakonov-Perel spin-relaxation times of $S_{x}$ and $S_{z}$ components in the presence of an electric field (Rashba effect) along the growth axis.

\section{SAMPLE PREPARATION AND CHARACTERIZATION}

The out-of-plane magnetic sample's (Device A) configuration is shown in Fig. 1(a). The quantum well $p-i-n$ structure and the tunnel barrier were grown by molecular beam epitaxy (MBE), while the spin filter structure was deposited by sputtering. The $p-i-n$ LED contains the following structure: $p$ - GaAs(001): $\mathrm{Zn}\left(p=2 \times 10^{19} \mathrm{~cm}^{-3}\right)$ substrate/ 500nm $p$-GaAs: $\mathrm{Be}\left(p=2.17 \times 10^{19} \mathrm{~cm}^{-3}\right) / 200$-nm $p$ - GaAs $\left(p=2 \times 10^{18} \mathrm{~cm}^{-3}\right) / 50 \mathrm{~nm}$ undoped GaAs/10-nm undoped $\mathrm{In}_{0.1} \mathrm{Ga}_{0.9} \mathrm{As} \mathrm{QW} / 50$-nm undoped GaAs/ 50-nm $n$-GaAs:Si $\left(n=1 \times 10^{16} \mathrm{~cm}^{-3}\right)$. The surface of the $p-i-n$ structure was passivated with arsenic in the III-V MBE chamber and then transferred through the air into another MBE-sputtering interconnected system. The arsenic capping layer was first desorbed at $300{ }^{\circ} \mathrm{C}$ by monitoring in situ reflection high energy electron diffraction patterns in the MBE chamber. Then the sample was transferred to the sputtering chamber without breaking the vacuum to grow $\mathrm{MgO}(2.5 \mathrm{~nm}) / \mathrm{Co}_{40} \mathrm{Fe}_{40} \mathrm{~B}_{20}$ $(1.1 \mathrm{~nm})$ layers at RT. Finally, 5-nm Ta was deposited to prevent oxidation, the $300-\mu \mathrm{m}$ diameter circular mesas and $100-\mu \mathrm{m}$ electrode $\mathrm{Au} / \mathrm{Ti}$ were then processed using standard UV photolithography and etching techniques. To achieve perpendicular magnetic anisotropy, the spin-LED device has been annealed with the rapid temperature annealing technique for a duration of $3 \mathrm{~min}$ [28-31]. The optical microscopy images, $I-V$ characteristics, and circularly polarized electroluminescence of Device A are shown in Fig. S1 in the Supplemental Material [32]. Device B [Fig. 1(d)] is based on an $\mathrm{AlGaAs} / \mathrm{GaAs} \mathrm{QW} / \mathrm{AlGaAs}$ LED structure with a $\mathrm{MgO}$ $(2.5 \mathrm{~nm}) / \mathrm{Co}_{40} \mathrm{Fe}_{40} \mathrm{~B}_{20}(3 \mathrm{~nm})$ in-plane magnetized spin filter [27].

A mode-locked Ti: Sapphire laser with a repetition rate of $80 \mathrm{MHz}$ and a pulse width of $140 \mathrm{fs}$ serves as the excitation source. The excitation wavelength is tuned from 700 to $970 \mathrm{~nm}$, which covers the excitation energy of the first valence subband of heavy holes to the first conduction subband (HH1e1) and the first valence subband of light holes to the first conduction subband (LH1-e1) transition in the $\mathrm{In}_{0.1} \mathrm{Ga}_{0.9} \mathrm{As}$ 

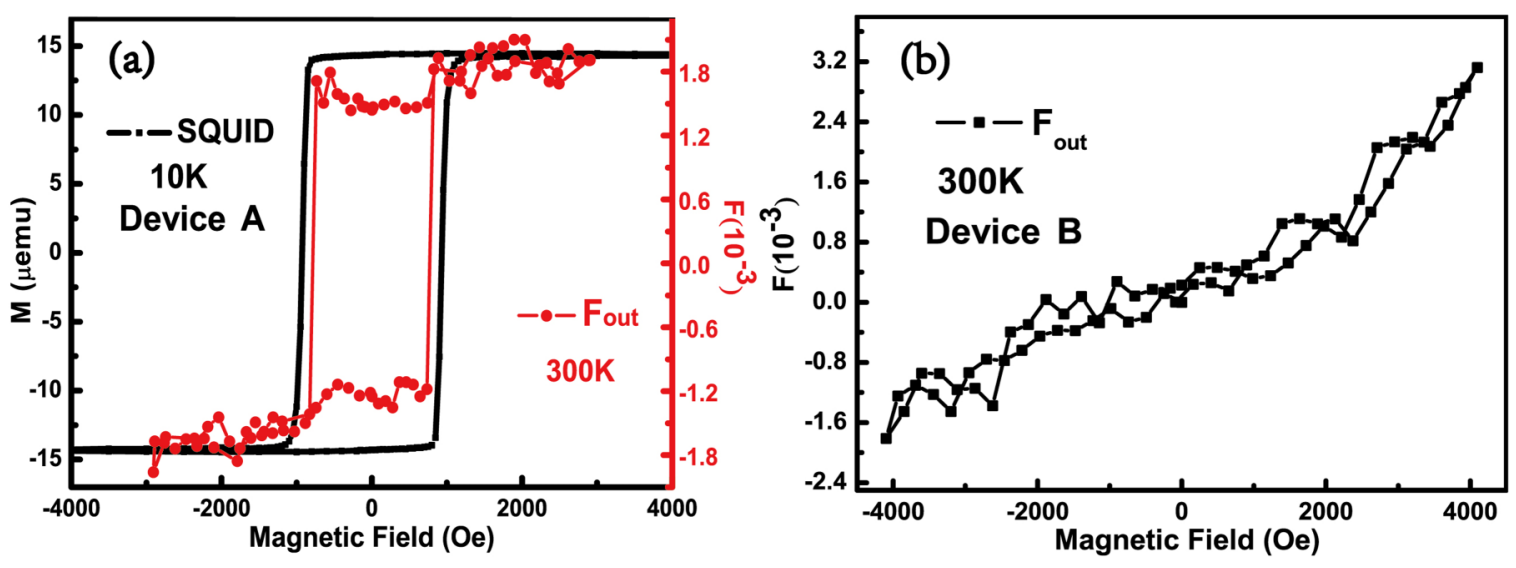

FIG. 2. The helicity asymmetry $F$ as a function of out-of-plane magnetic field measured at $300 \mathrm{~K}$ in Devices A and B. (a) The out-of-plane magnetization helicity asymmetry $F_{\text {out }}$ compared to the corresponding out-of-plane $M$ - $H$ hysteresis loop measured at $10 \mathrm{~K}$ by SQUID in Device A. (b) The helicity asymmetry $F_{\text {out }}$ measured in Device B.

QW of Device A and the GaAs QW of Device B. The incident light goes through a polarizer and a photo elastic modulator whose retardation is set to be $0.25 \lambda$ to yield a modulated circularly polarized light with a fixed modulating frequency at $50 \mathrm{KHz}$, and the spin-polarized photocurrent $(\Delta I)$ is first amplified by a current preamplifier and then collected by a lock-in amplifier with the reference frequency of $50 \mathrm{KHz}$. A chopper with a frequency at $220 \mathrm{~Hz}$ is applied to produce a polarization independent light, which is used to measure the common photoinduced direct current $\left(I_{p h}\right)$ that is proportional to the total number of photoinduced electrons, and $I_{p h}$ is also first amplified by a current preamplifier and then collected by a lock-in amplifier with the reference frequency of $220 \mathrm{~Hz}$. We define the photocurrent helicity asymmetry $F$ (figure of merit) as $F=\Delta I / I_{p h}$. For the investigation of Device A (outof-plane spin filter), the excitation light propagates perpendicularly to the layers [normal incidence, Fig. 1(b)] whereas for the measurements of Device B, the oblique incidence is used with circularly polarized light [Fig. 1(c)]. As is shown in Fig. 1, the observed photocurrent asymmetry results from the spin filtering effect of electron $S_{x}$ and $S_{z}$ components by inplane and out-of-plane magnetized $\mathrm{CoFeB}$ layer, respectively. Under these conditions, the helicity asymmetry in the out-ofplane and in-plane magnetic field are expressed as $F_{\text {out }}$ and $F_{\text {in }}$, respectively. The measured $\Delta I$ for Device $\mathrm{A}$ are presented in Figs. S2, S3 in the Supplemental Material.

\section{RESULTS AND DISCUSSION}

\section{A. Magnetic field dependence}

In order to have a detailed understanding of the origin of the helicity asymmetry, we have performed a study of $F$ variations at different magnetic fields at $300 \mathrm{~K}$. Both Devices $A$ and $B$ were placed into a Helmholtz split magnetic coil providing a magnetic field perpendicular to the sample plane. The excitation wavelength is fixed at the energy of the LH1-e1 transition in the quantum well at $300 \mathrm{~K}$ : for Device B (GaAs QW), this corresponds to $825 \mathrm{~nm}$ and for Device A $\left(\operatorname{In}_{0.1} \mathrm{Ga}_{0.9} \mathrm{As} \mathrm{QW}\right)$, the wavelength is $925 \mathrm{~nm}$. Figure 2(a) demonstrates clearly that the helicity asymmetry of the photocurrent can be evidenced in Device A (perpendic- ularly magnetized electrode) for zero external magnetic field and at room temperature. The helicity asymmetry $F_{\text {out }}$ exhibits a clear hysteresis loop feature, with an almost constant value around $0.2 \%$ at saturation and changes its sign rapidly at $\mathrm{H}= \pm 800$ Oe. Figure 2(a) also displays out-of-plane $M-H$ hysteresis measured at $10 \mathrm{~K}$ by superconducting quantum interference device (SQUID). We note that the photocurrent helicity asymmetry hysteresis loop measured at $300 \mathrm{~K}$ follows well the $M-H$ hysteresis loop at $10 \mathrm{~K}$. Although the out-ofplane coercivity is reduced with increasing the temperature [33], it is sufficient to maintain an almost full remanent magnetization at $300 \mathrm{~K}$.

For comparison, we have measured the variation of the photocurrent helicity asymmetry in Device B (in-plane magnetized electrode) in the same conditions (normal incidence and magnetic field applied perpendicular to the layers), as shown in Fig. 2(b). As expected, no spin dependent photocurrent $\left(F_{\text {out }}=0\right)$ is observed at zero field (in contrast to Device A). This can be understood because the in-plane spin filter cannot detect the photogenerated $S_{z}$ component of the electron spin. We observe that $F_{\text {out }}$ increases quasilinearly with the magnetic field, following the increasing of the out-of-plane component of the magnetization. Indeed, in this configuration, the helicity asymmetry $F_{\text {out }}$ tracks the continuous rotation of the magnetization direction from in-plane to out-of-plane. However, as shown in Ref. [33], the interpretation of the origin of the photocurrent asymmetry in these conditions for Device $\mathrm{B}$ is much more complicated since the Zeeman splitting of the electronic states in the QW (due to the external magnetic field) may yield different absorption coefficients for right or left circularly polarized light.

\section{B. Bias dependence}

We have performed a systematic measurement of the spectra $\Delta I$ and $I_{p h}$ as a function of the bias voltage at $77 \mathrm{~K}$ for Device A. Then the spectra of helicity asymmetry $F_{\text {out }}$ have been deduced at $77 \mathrm{~K}$ [see Fig. 3(b)]. In Figs. 3(a) and 3 (b), we identify clearly the energy positions corresponding to the optical transitions in the InGaAs QW at approximately $875 \mathrm{~nm}(77 \mathrm{~K})$. It corresponds fairly well to the heavy-hole- 

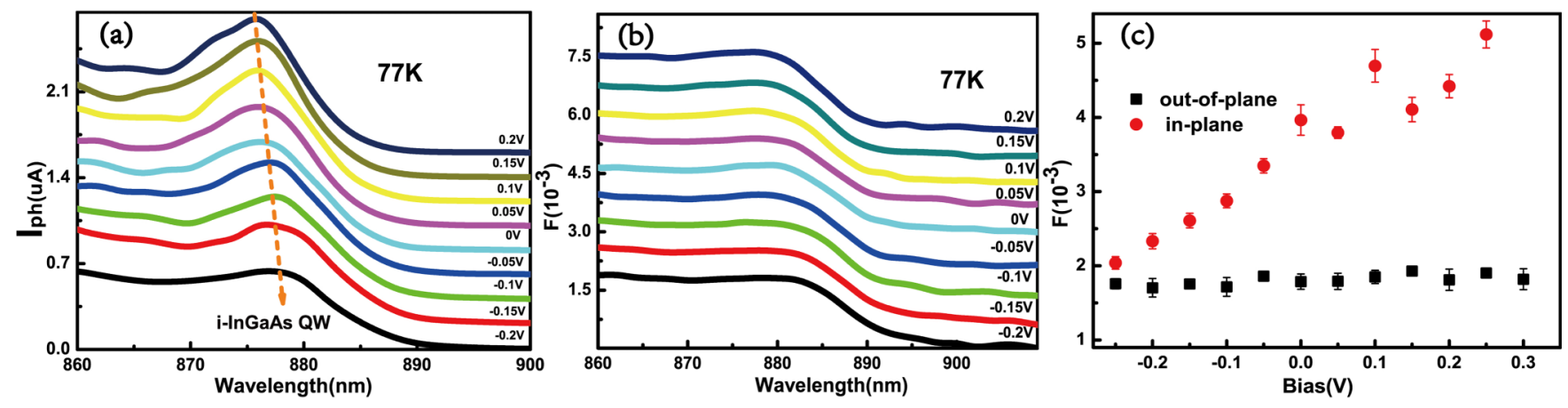

FIG. 3. Bias voltage dependence of the spectra of total photocurrents and helicity asymmetry for Device A (out-of-plane magnetization) at $77 \mathrm{~K}$. (a) Spectra of the total photoinduced current. (b) Spectra of the helicity asymmetry at different bias voltages at $77 \mathrm{~K}$. Note that the spectra in (a) and (b) are intentionally shifted for clarity. (c) The helicity asymmetry $F_{\text {out }}$ in Device A [deduced from Fig. 3(b)] as a function of bias voltage. It is compared to the helicity asymmetry $F_{\text {in }}$ in Device B measured in Ref. [27], where the incident angle in these experiments is fixed at $15^{\circ}$.

electron transitions calculated with a six-band $\mathbf{k} \cdot \mathbf{p}$ model. It also demonstrates that the asymmetry is at its maximum close to the InGaAs quantum well optical transitions. With the voltage varying from +0.3 to $-0.25 \mathrm{~V}$, the $I_{p h}$ peak positions corresponding to the InGaAs QW transition reveal a clear red shift corresponding to the quantum confined Stark effect [34] due to the electric field in the device [see Fig. 3(a)]. The influence of the bias on the helicity asymmetry $F$ related to the quantum well is shown in Fig. 3(b). In Fig. 3(c), we show the helicity asymmetry measured at the wavelength corresponding to the quantum-well absorption maximum as a function of bias. Remarkably, we observe that the asymmetry of the spin photocurrent in Device A does not depend on the applied bias within our uncertainty. This is in contrast with the strong bias dependence of the helicity asymmetry measured in Device B (in-plane magnetization), as shown with circles in Fig. 3(c). Though a quantitative comparison is not possible (due to the differences of measurement geometry, spin filter, and LED structures in the two devices), we believe that the strong difference in the bias dependence of the photocurrent helicity asymmetry is a direct consequence of the large spin relaxation anisotropy in the III-V quantum well of the device.

First let us recall that the amplitude of the photocurrent helicity asymmetry $F_{\text {out }}$ depends on the relaxation of the electron $S_{z}$ spin component in Device A (out-of-plane magnetization, normal optical incidence) and $F_{\text {in }}$ depends on the relaxation of the electron $S_{x}$ spin component in Device B (in-plane magnetization, oblique incidence, Ref. [27]). In the presence of an external electric field $\boldsymbol{E}$, the electron spin relaxation in a III-V QW is controlled by the Dyakonov-Perel spin relaxation governed by the interplay between the bulk inversion asymmetry (Dresselhaus contribution) and the structural inversion asymmetry (Rashba contribution due to the presence of the electric field) [35-38].

For the (001) grown quantum wells present in our devices, the effective magnetic field due to the Dresselhaus term, $B_{\mathrm{D}}(\boldsymbol{k})$ or due the Rashba term $B_{\mathrm{R}}(\boldsymbol{k})$ lies in the quantum well plane $x y$ ( $\boldsymbol{k}$ is the electron wave vector); its amplitude and
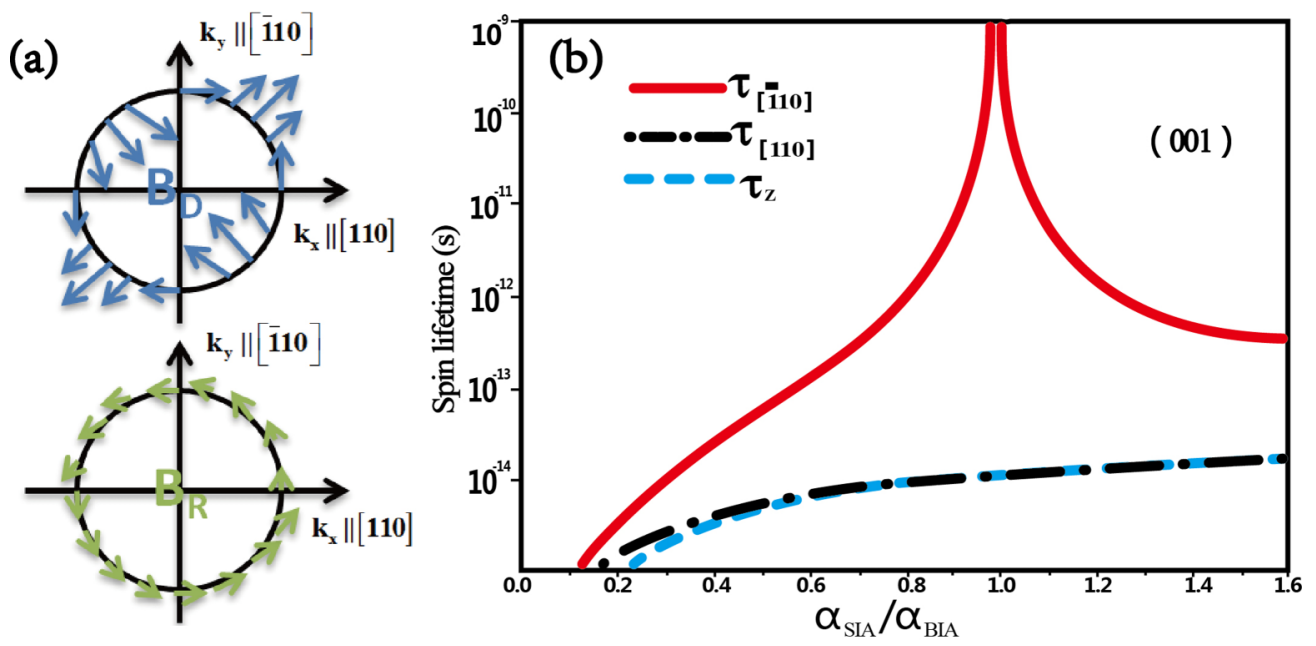

FIG. 4. (a) Orientations of the spin-orbit effective magnetic fields associated with the bulk inversion asymmetry [Dresselhaus contribution, $B_{\mathrm{D}}(\boldsymbol{k})$ ] and with the structural inversion asymmetry [Rashba contribution, $B_{\mathrm{R}}(\boldsymbol{k})$ ] in III-V quantum wells (QWs) with (001) orientations. (b) Dependence of the spin relaxation time of $S_{x}\left(\tau_{[-110]}\right), S_{y}\left(\tau_{[110]}\right)$, and $S_{z}\left(\tau_{z}\right)$ components as a function of the electric field that controls the Rashba term; the large increase of the $S_{x}$ relaxation time occurs when the Rashba and Dresselhaus contributions compensate each other [see Eq. (S1) in the Supplemental Material]. 

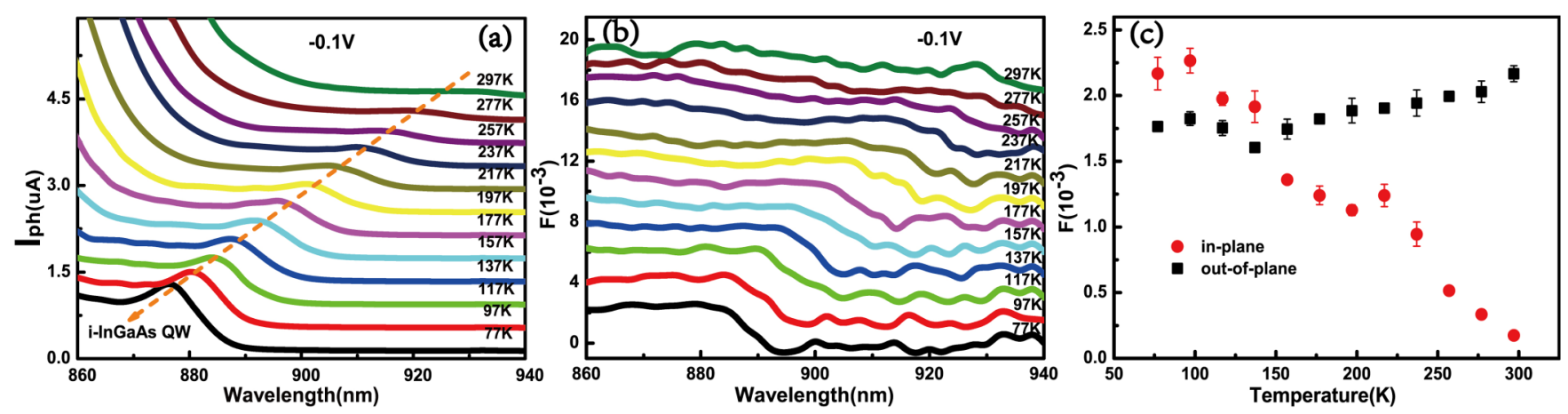

FIG. 5. Temperature dependence of the spectra of total photocurrents and helicity asymmetry for Device A at $-0.1 \mathrm{~V}$ bias. (a) Spectra of the total photoinduced direct currents. (b) Spectra of the helicity asymmetry $F_{\text {out }}$ at different temperature at $-0.1 \mathrm{~V}$. Note that the spectra in (a) and (b) are intentionally shifted for clarity. (c) The helicity asymmetry $F_{\text {in }}$ (Device B, from Ref. [27]) and $F_{\text {out }}$ as a function of temperature.

orientation depending on $\boldsymbol{k}$, see Fig. 4(a). This yields very different dependences of the electron spin relaxation times $S_{x}, S_{y}$ or $S_{z}$ [see Fig. 4(b)] as a function of the electric field. As shown in Fig. 4(b), the spin relaxation time of the $S_{z}$ component $\left(\tau_{z}\right)$ depends very weakly on the amplitude of the electric field. This is perfectly consistent with the almost constant $F_{\text {out }}$ measured in Device A [Fig. 3(c)]. In contrast, the spin relaxation time of the $S_{x}$ component $\left(\tau_{[-110]}\right)$ exhibits a very strong electric field dependence. In Device B, the photocurrent helicity asymmetry likely depends on the relaxation of both $S_{x}$ and $S_{z}$ components. Though $S_{z}$ weakly depends on the electric field, the overall effect will be a significant dependence with the bias, as observed in Fig. 3(c) for Device B. From the doping concentration and the thickness of the layers, it is possible to estimate an electric field in our devices $E \sim 100 \mathrm{kV} / \mathrm{cm}$. This typical value is in the region where the spin relaxation time of $S_{x}$ component varies strongly with $E$ [36].

\section{Temperature dependence}

The temperature dependence of the spectra of $I_{p h}$ and the deduced helicity asymmetry $F_{\text {out }}$ in Device A with a fixed bias of $-0.1 \mathrm{~V}$ are shown in Figs. 5(a) and 5(b), respectively. We extract the temperature dependence of the peak position of the helicity asymmetry corresponding to the optical transition of InGaAs QW. The energy corresponding to the InGaAs QW shows a red shift with the increase of the temperature, which is simply due to the temperature dependence of the band gap. The temperature dependence of the helicity asymmetry of the photocurrent can be extracted [Fig. 5(c)]. The striking feature is that the helicity asymmetry $F_{\text {out }}$ does not vary much from 77 to $300 \mathrm{~K}$ for Device A. In contrast, $F_{\text {in }}$ of Device B decreases from $0.2 \%$ down to zero in the same temperature range.

The temperature dependence of $F$ likely reflects physical effects inherent to the semiconductor heterostructures rather than to the property of the CoFeB magnetic layer itself. $F$ mainly depends on the spin relaxation rate and the electron lifetime in the QW $[31,39,40]$. As the QW in the two devices have different characteristics $\left(\mathrm{In}_{0.1} \mathrm{GaA}_{0.9} \mathrm{As} / \mathrm{GaAs} \mathrm{QW}\right.$ for Device A and GaAs $/ \mathrm{Al}_{0.08} \mathrm{Ga}_{0.92} \mathrm{As}$ QW for Device B), it is rather speculative to interpret the change of temperature variations. Nevertheless, the dependence in Device A and B could be also related to the variation of the Dyakonov-Perel spin relaxation time of the $S_{z}$ and $S_{x}$ components, respectively. To the best of our knowledge, the differences of these spin relaxation times have never been investigated experimentally. But from a theoretical point of view, no drastic change is expected [41,42]. Further work is required to elucidate this point.

\section{CONCLUSIONS}

We have detected at room temperature photogenerated spin photocurrents by using an out-of-plane magnetic electrode without the need for an external magnetic field. The bias and temperature dependences are measured and compared with those of a spin photocurrent device with in-plane $\mathrm{CoFeB} / \mathrm{MgO}$ spin filter and are excited under oblique incidence with circularly polarized light. We show that the differences in the two devices' characteristics are linked to the different orientation of electron spin filtered by the $\mathrm{CoFeB}$ electrode and interpreted on the basis of the Dyakonov-Perel spin-relaxation time and the giant spin relaxation anisotropy in III-V QWs in the presence of an electric field. Our research also provides a method to study the spin relaxation anisotropy in QWs. This demonstration of a stable helicity asymmetry of photocurrent resulting from an out-of-plane magnetic electrode paves the way for future applications based on circularly polarized light detection via photocurrent in spinoptronic devices working at room temperature.

\section{ACKNOWLEDGMENTS}

The work is supported by National Key Research and Development Program (Grants No. 2016YFB0402303 and No. 2018YFA0209103), the National Basic Research Program of China (Grant No. 2015CB921503), the National Natural Science Foundation of China (Grants No. 11574302, No. 61474114, No. 61627822, and No. 11704032). X.M. acknowledges the Institut Universitaire de France. P.R. acknowledges the Nano Grant No. ANR-17-EURE-0009 in the framework of the Programme des Investissements d'Avenir. Y.L. acknowledges the joint French National Research AgencyNational Natural Science Foundation of China SISTER project (Grants No. ANR-11-IS10-0001 and No. NNSFC 61161130527) and the ENSEMBLE project (Grants No. ANR-14-0028-01 and No. NNSFC 61411136001). 
[1] I. Zutic, J. Fabian, and S. Das Sarma, Rev. Mod. Phys. 76, 323 (2004).

[2] S. A. Wolf, D. D. Awschalom, R. A. Buhrman, J. M. Daughton, S. von Molnar, M. L. Roukes, A. Y. Chtchelkanova, and D. M. Treger, Science 294, 1488 (2001).

[3] D. D. Awschalom, M. E. Flatte, and N. Samarth, Sci. Am. 286, 66 (2002).

[4] M. Holub and P. Bhattacharya, J. Phys. D 40, R179 (2007).

[5] R. Fiederling, M. Keim, G. a. Reuscher, W. Ossau, G. Schmidt, A. Waag, and L. Molenkamp, Nature (London) 402, 787 (1999).

[6] Y. Ohno, D. K. Young, B. Beschoten, F. Matsukura, H. Ohno, and D. D. Awschalom, Nature (London) 402, 790 (1999).

[7] T. D. Nguyen, E. Ehrenfreund, and Z. V. Vardeny, Science 337, 204 (2012).

[8] V. G. Truong, P. H. Binh, P. Renucci, M. Tran, Y. Lu, H. Jaffres, J. M. George, C. Deranlot, A. Lemaitre, T. Amand, and X. Marie, Appl. Phys. Lett. 94, 141109 (2009).

[9] R. Farshchi, M. Ramsteiner, J. Herfort, A. Tahraoui, and H. T. Grahn, Appl. Phys. Lett. 98, 162508 (2011).

[10] E. J. Gansen, K. Jarasiunas, and A. L. Smirl, Appl. Phys. Lett. 80, 971 (2002).

[11] P. Asshoff, A. Merz, H. Kalt, and M. Hetterich, Appl. Phys. Lett. 98, 112106 (2011).

[12] J. Xu, A. Lakhtakia, J. Liou, A. Chen, and I. J. Hodgkinson, Opt. Commun. 264, 235 (2006).

[13] D.-Y. Kim, J. Korean Phys. Soc. 49, S505 (2006).

[14] S. A. Crooker, M. Furis, X. Lou, C. Adelmann, D. L. Smith, C. J. Palmstrom, and P. A. Crowell, Science 309, 2191 (2005).

[15] S. A. Crooker and D. L. Smith, Phys. Rev. Lett. 94, 236601 (2005).

[16] M. Cantoni and C. Rinaldi, J. Appl. Phys. 120, 104505 (2016).

[17] R. C. Roca, N. Nishizawa, K. Nishibayashi, and H. Munekata, Jpn. J. Appl. Phys. 56, 04CN05 (2017).

[18] T. Taniyama, G. Wastlbauer, A. Ionescu, M. Tselepi, and J. A. C. Bland, Phys. Rev. B 68, 134430 (2003).

[19] A. F. Isakovic, D. M. Carr, J. Strand, B. D. Schultz, C. J. Palmstrøm, and P. A. Crowell, Phys. Rev. B 64, 161304(R) (2001).

[20] F. Meier and B. P. Zakharchenya, Optical Orientation (NorthHolland, Amsterdam, 1987).

[21] M. I. Dyakonov, Spin Physics in Semiconductors (Springer, Berlin, 2008).

[22] C. Adelmann, X. Lou, J. Strand, C. J. Palmstrøm, and P. A. Crowell, Phys. Rev. B 71, 121301(R) (2005).

[23] X. Y. Dong, C. Adelmann, J. Q. Xie, C. J. Palmstrom, X. Lou, J. Strand, P. A. Crowell, J. P. Barnes, and A. K. Petford-Long, Appl. Phys. Lett. 86, 102107 (2005).

[24] N. Nishizawa and H. Munekata, J. Appl. Phys. 114, 033507 (2013).
[25] P. Renucci, V. G. Truong, H. Jaffres, L. Lombez, P. H. Binh, T. Amand, J. M. George, and X. Marie, Phys. Rev. B 82, 195317 (2010).

[26] S. Hövel, N. C. Gerhardt, M. R. Hofmann, F.-Y. Lo, A. Ludwig, D. Reuter, A. D. Wieck, E. Schuster, H. Wende, W. Keune, O. Petracic, and K. Westerholt, Appl. Phys. Lett. 93, 021117 (2008).

[27] L. Zhu, W. Huang, P. Renucci, X. Marie, Y. Liu, Y. Li, Q. Wu, Y. Zhang, B. Xu, Y. Lu, and Y. Chen, Phys. Rev. Appl. 8, 064022 (2017).

[28] B. S. Tao, P. Barate, J. Frougier, P. Renucci, B. Xu, A. Djeffal, H. Jaffres, J. M. George, X. Marie, S. Petit-Watelot, S. Mangin, X. F. Han, Z. G. Wang, and Y. Lu, Appl. Phys. Lett. 108, 152404 (2016).

[29] I. Ozfidan, M. Korkusinski, A. D. Guclu, J. A. McGuire, and P. Hawrylak, Phys. Rev. B 89, 085310 (2014).

[30] P. Barate, S. Liang, T. T. Zhang, J. Frougier, M. Vidal, P. Renucci, X. Devaux, B. Xu, H. Jaffres, J. M. George, X. Marie, M. Hehn, S. Mangin, Y. Zheng, T. Amand, B. Tao, X. F. Han, Z. Wang, and Y. Lu, Appl. Phys. Lett. 105, 012404 (2014).

[31] S. H. Liang, T. T. Zhang, P. Barate, J. Frougier, M. Vidal, P. Renucci, B. Xu, H. Jaffrès, J. M. George, X. Devaux, M. Hehn, X. Marie, S. Mangin, H. X. Yang, A. Hallal, M. Chshiev, T. Amand, H. F. Liu, D. P. Liu, X. F. Han, Z. G. Wang, and Y. Lu, Phys. Rev. B 90, 085310 (2014).

[32] See Supplemental Material at http://link.aps.org/supplemental/ 10.1103/PhysRevB.100.045417 for the optical microscopy images, $I-V$ characteristics, and circularly polarized electroluminescence of Device A.

[33] J. Stöhr, Magnetism: From Fundamentals to Nanoscale Dynamics (Springer, Berlin, 2006).

[34] H. Q. Le, J. J. Zayhowski, and W. D. Goodhue, Appl. Phys. Lett. 50, 1518 (1987).

[35] D. Stich, J. H. Jiang, T. Korn, R. Schulz, D. Schuh, W. Wegscheider, M. W. Wu, and C. Schuller, Phys. Rev. B 76, 073309 (2007).

[36] A. Balocchi, Q. H. Duong, P. Renucci, B. L. Liu, C. Fontaine, T. Amand, D. Lagarde, and X. Marie, Phys. Rev. Lett. 107, 136604 (2011).

[37] I. Vurgaftman and J. R. Meyer, J. Appl. Phys. 97, 053707 (2005).

[38] N. S. Averkiev, L. E. Golub, and M. Willander, J Phys.: Condens. Mater. 14, R271 (2002).

[39] A. Malinowski, R. S. Britton, T. Grevatt, R. T. Harley, D. A. Ritchie, and M. Y. Simmons, Phys. Rev. B 62, 13034 (2000).

[40] R. Wang, X. Jiang, R. M. Shelby, and S. S. P. Parkin, Appl. Phys. Lett. 87, 262503 (2005).

[41] M. D'yakonov and V. Perel, J. Exp. Theor. Phys 33, 1053 (1971).

[42] M. I. Dyakonov and V. I. Perel, Sov. Phys. Solid State 13, 3023 (1972). 